\title{
Трехмерные метрические группы Ли с векторным кручением и нулевым тензором кривизны
}

\author{
С.В. Клепикова, И.В. Эрнст
}

Алтайский государственный университет (Барнаул, Россия)

\section{Three-Dimensional Metric Lie Groups with Vectorial Torsion and Zero Curvature Tensor}

\author{
S.V. Klepikova, I.V. Ernst \\ Altai State University (Barnaul, Russia)
}

Последнее время становится актуальным изучение (псевдо)римановых многообразий с различными метрическими связностями, отличными от связности Леви-Чивита.

Метрическая связность с векторным кручением (также известная как полусимметрическая связность) является одной из часто рассматриваемых связностей.

Связь между конформными деформациями римановых многообразий и метрическими связностями с векторным кручением на них была установлена в работах К. Яно.

А именно: риманово многообразие допускает метрическую связность с векторным кручением, тензор кривизны которой равен нулю, тогда и только тогда, когда оно является конформно плоским.

Кроме того, данная связность играет важную роль в случае двумерных поверхностей, так как в этом случае любая метрическая связность является связностью с векторным кручением.

Таким образом, возникает задача об изучении (псевдо)римановых многообразий с метрической связностью с векторным кручением, тензор кривизны которых равен нулю.

Данная работа посвящена решению поставленной задачи в случае трехмерных метрических групп Ли. Кроме того, приводится математическая модель, позволяющая вычислять компоненты тензора кривизны метрической связности с векторным кручением в случае метрических групп Ли.

Ключевые слова: (псевдо)римановое многообразие, метрическая связность с векторным кручением, группы Ли, тензор кривизны.

DOI 10.14258/izvasu(2019)4-14

1. Введение, определения и постановка задачи. Пусть $(M, g)$ - (псевдо)риманово многообразие. Определим на данном многообразии

* Работа выполнена при поддержке РФФИ (грант: № 18 31-00033 мол_а).
Recently, the study of (pseudo)Riemannian manifolds with different metric connections different from the LeviCivita connection becomes relevant.

A metric connection with vectorial torsion (also known as a semi-symmetric connection) is one of the often considered connections.

The connection between the conformal deformations of Riemannian manifolds and metric connections with vectorial torsion on them was established in the works of K. Yano.

Namely, a Riemannian manifold admits a metric connection with vectorial torsion, the curvature tensor of which is zero, if and only if it is conformally flat.

Moreover, this connection plays an important role in the case of two-dimensional surfaces since, in this case, any metric connection is a connection with vectorial torsion.

Thus, the problem of studying (pseudo)Riemannian manifolds with metric connection with vectorial torsion, the curvature tensor of which is zero, is arisen.

This paper is devoted to solving the problem in the case of three-dimensional metric Lie groups. In addition, a mathematical model is presented that allows one to calculate the components of the curvature tensor of a metric connection with vectorial torsion in the case of metric Lie groups.

Key words: (pseudo)Riemannian manifold, metric connection with vectorial torsion, Lie groups, curvature tensor.

метрическую связность $\nabla$ при помощи равенства

$$
\nabla_{X} Y=\nabla_{X}^{g} Y+g(X, Y) V-g(V, Y) X,
$$

где $V$ - некоторое фиксированное векторное поле, $X$ и $Y-$ произвольные векторные поля, $\nabla^{g}-$ связность Леви-Чивита. Связность $\nabla$ называется метрической связностью с векторным круче- 
нием и является одной из трех основных связностей, описанных Э. Картаном в работе [1]. Также связность $\nabla$ называют полусимметрической связностью (с точностью до направления).

Данная связность играет важную роль в случае двумерных поверхностей, поскольку любая метрическая связность является связностью с векторным кручением в этом случае [1]. В работах [2-7] изучаются различные аспекты метрических связностей с векторным кручением.

В работе К. Яно [8] была сформулирована и доказана важная теорема о связи конформных деформаций и метрических связностей с векторным кручением.

Теорема. Риманово многообразие допускает метрическую связность с векторным кручением, тензор кривизны которой равен нулю, тогда и только тогда, когда оно является конформно плоским.

Таким образом, возникает задача об изучении (псевдо)римановых многообразий с метрической связностью с векторным кручением, тензор кривизны которых равен нулю. Данная работа посвящена решению поставленной задачи в случае трехмерных метрических групп Ли.

2. Предварительные сведения. Тензор кривизны метрической связности $\nabla$ с векторным кручением определяется аналогично общему случаю равенством:

$$
R(X, Y) Z=\nabla_{Y} \nabla_{X} Z-\nabla_{X} \nabla_{Y} Z+\nabla_{[X, Y]} Z .
$$

Отметим, что, в отличие от случая связности Леви-Чивита, в данном случае тензор кривизны не обязан удовлетворять алгебраическому тождеству Бьянки.

Исследование кривизны трехмерных метрических групп Ли основывается на следующей теореме, которая была доказана в римановом случае в работе [9], а в лоренцевом - в [10].

Теорема. Пусть $(M, g)$ - трехмерное локально однородное (псевдо)риманово многообразие. Тогда либо $(M, g)$ является локально симметричным (относительно связности Леви-Чивита), либо оно локально изометрично трехмерной группе Ли с левоинвариантной (псевдо)римановой метрикой.

Следующая классификация для трехмерных метрических групп Ли была получена в римановом случае в статье [11], а в лоренцевом в $[10,12,13]$.

Теорема 1. Пусть $G$ - трехмерная метрическая группа Ли, тогда соответствующая метрическая алгебра Ли содержится в таблице.

Опишем математическую модель, позволяющую вычислять компоненты тензора кривизны для метрических групп Ли с метрической связностью с инвариантным векторным кручением. За- фиксируем некоторый базис $\left\{e_{1}, e_{2}, \ldots, e_{n}\right\}$ в соответствующей алгебре Ли g. Положим,

$$
\left[e_{i}, e_{j}\right]=c_{i j}^{k} u_{e}, \quad\left\langle u_{i}, u_{j}\right\rangle=g_{i j},
$$

где $c_{i j}^{k}$ - структурные константы алгебры Ли, $g_{i j}$ - компоненты метрического тензора.

Компоненты связности Леви-Чивита $\nabla^{g}$ выражаются через структурные константы и компоненты метрического тензора:

$$
\left(\Gamma^{g}\right)_{i j}^{k}=\frac{1}{2}\left(c_{i j}^{k}+g^{s k} c_{s j}^{l} g_{i l}+g^{s k} c_{s i}^{l} g_{j l}\right),
$$

где $\nabla_{e_{i}}^{g} e_{j}=\left(\Gamma^{g}\right)_{i j}^{k} e_{k}$ и $\left\{g^{i j}\right\}$ - матрица, обратная к матрице $\left\{g_{i j}\right\}$.

Пусть инвариантный вектор $V \in \mathfrak{g}$, тогда компоненты метрической связности $\nabla$ с векторным кручением (1) задаются равенствами:

$$
\Gamma_{i j}^{k}=\left(\Gamma^{g}\right)_{i j}^{k}+g_{i j} V^{k}-V^{s} g_{s j} \delta_{i}^{k},
$$

где $\nabla_{e_{i}} e_{j}=\Gamma_{i j}^{k} e_{k}$.

Компоненты тензора кривизны $R$ можно вычислить с помощью следующей формулы:

$$
R_{i j k s}=\left(\Gamma_{i k}^{l} \Gamma_{j l}^{p}-\Gamma_{j k}^{l} \Gamma_{i l}^{p}+c_{i j}^{l} \Gamma_{l k}^{p}+C_{i j}^{l} \bar{\Gamma}_{l k}^{p}\right) g_{p s} .
$$

3. Основной результат. Главным результатом данной работы является следующая

Теорема 2. Пусть $(G, g)$ - трехмерная метрическая группа Ли с метрической связностью с инвариантным векторным кручением. Тогда если тензор кривизны равен нулю, то

- в случае $\mathcal{U}: V=0$ и

$$
\begin{aligned}
& \text { 1. либо } \alpha_{1}=0, \alpha_{2}=\alpha_{3} ; \\
& \text { 2. либо } \alpha_{2}=0, \alpha_{1}=\alpha_{3} ; \\
& \text { 3. либо } \alpha_{3}=0, \alpha_{1}=\alpha_{2} ;
\end{aligned}
$$

- в случае $\mathcal{N U}$ :

$$
\begin{aligned}
& \text { 1. либо } \alpha_{2}=1 \pm \sqrt{1-\alpha_{1}^{2}}, \alpha_{1}=\alpha_{3}, \\
& V=(-2,0,0) ; \\
& \text { 2. либо } \alpha_{2}=1 \pm \sqrt{1-\alpha_{1}^{2}}, \alpha_{1}=\alpha_{3}, \\
& V=\left(0,-\frac{\left(1 \pm \sqrt{1-\alpha_{1}^{2}}\right) V^{3}}{\alpha_{1}}, V^{3}\right), \quad \text { где } \\
& V^{3}=\sqrt{ \pm 2 \sqrt{1-\alpha_{1}^{2}}-2}
\end{aligned}
$$

- в случае $\mathcal{A}_{1}: V=0$ и

$$
\begin{aligned}
& \text { 1. либо } \alpha_{1}=0, \alpha_{2}=\alpha_{3} ; \\
& \text { 2. либо } \alpha_{2}=0, \alpha_{1}=-\alpha_{3} ; \\
& \text { 3. либо } \alpha_{3}=0, \alpha_{1}=-\alpha_{2} ;
\end{aligned}
$$

- в случае $\mathcal{A}_{2}: \alpha_{1}=0, \alpha_{2}=0$ и $V=0$;

- в случае $\mathcal{A}_{3}: \alpha_{1}=0$ и

1. либо $V=(0,1,1)$;

2. либо $V=(0,-2,-2)$; 
Трехмерные метрические алгебры Ли

\begin{tabular}{|c|c|c|c|}
\hline Случай & Скобки Ли & $\begin{array}{c}\text { Нетривиальные } \\
\text { скалярные произведения }\end{array}$ & Ограничения \\
\hline \multicolumn{4}{|c|}{ Риманова метрика } \\
\hline $\mathcal{U}$ & {$\left[e_{1}, e_{2}\right]=\alpha_{3} e_{3},\left[e_{1}, e_{3}\right]=-\alpha_{2} e_{2},\left[e_{2}, e_{3}\right]=\alpha_{1} e_{1}$} & \multirow{2}{*}{$\begin{array}{c}\left\langle e_{1}, e_{1}\right\rangle=1,\left\langle e_{2}, e_{2}\right\rangle=1 \\
\left\langle e_{3}, e_{3}\right\rangle=1\end{array}$} & - \\
\hline $\mathcal{N U}$ & $\begin{array}{c}{\left[e_{1}, e_{2}\right]=\left(2-\alpha_{2}\right) e_{2}+\alpha_{3} e_{3},\left[e_{1}, e_{3}\right]=\alpha_{1} e_{2}+\alpha_{2} e_{3},} \\
{\left[e_{2}, e_{3}\right]=0}\end{array}$ & & - \\
\hline \multicolumn{4}{|c|}{ Лоренцева метрика } \\
\hline $\mathcal{A}_{1}$ & {$\left[e_{1}, e_{2}\right]=\alpha_{3} e_{3},\left[e_{1}, e_{3}\right]=-\alpha_{2} e_{2},\left[e_{2}, e_{3}\right]=\alpha_{1} e_{1}$} & $\begin{array}{c}\left\langle e_{1}, e_{1}\right\rangle=-1,\left\langle e_{2}, e_{2}\right\rangle=1 \\
\left\langle e_{3}, e_{3}\right\rangle=1\end{array}$ & - \\
\hline $\mathcal{A}_{2}$ & $\begin{array}{c}{\left[e_{1}, e_{2}\right]=\left(1-\alpha_{2}\right) e_{3}-e_{2},\left[e_{1}, e_{3}\right]=e_{3}-\left(1+\alpha_{2}\right) e_{2}} \\
{\left[e_{2}, e_{3}\right]=\alpha_{1} e_{1}}\end{array}$ & \multirow{2}{*}{$\begin{array}{c}\left\langle e_{1}, e_{1}\right\rangle=1,\left\langle e_{2}, e_{2}\right\rangle=1 \\
\left\langle e_{3}, e_{3}\right\rangle=-1\end{array}$} & - \\
\hline $\mathcal{A}_{3}$ & $\begin{aligned} {\left[e_{1}, e_{2}\right]=} & e_{1}-\alpha_{1} e_{3},\left[e_{1}, e_{3}\right]=-\alpha_{1} e_{2}-e_{1}, \\
& {\left[e_{2}, e_{3}\right]=\alpha_{1} e_{1}+e_{2}+e_{3} }\end{aligned}$ & & - \\
\hline $\mathcal{A}_{4}$ & $\begin{array}{c}{\left[e_{1}, e_{2}\right]=\alpha_{3} e_{2},\left[e_{1}, e_{3}\right]=-\alpha_{2} e_{1}-\alpha_{1} e_{2},} \\
{\left[e_{2}, e_{3}\right]=-\alpha_{1} e_{1}+\alpha_{2} e_{2}}\end{array}$ & $\begin{array}{c}\left\langle e_{1}, e_{1}\right\rangle=-1,\left\langle e_{2}, e_{2}\right\rangle=1 \\
\left\langle e_{3}, e_{3}\right\rangle=1\end{array}$ & $\alpha_{2} \neq 0$ \\
\hline $\mathcal{A}$ & $\begin{array}{c}{\left[e_{1}, e_{2}\right]=0,\left[e_{1}, e_{3}\right]=\alpha_{1} \sin \alpha_{3} e_{1}-\alpha_{2} \cos \alpha_{3} e_{2}} \\
{\left[e_{2}, e_{3}\right]=\alpha_{1} \cos \alpha_{3} e_{1}+\alpha_{2} \sin \alpha_{3} e_{2}}\end{array}$ & $\begin{array}{c}\left\langle e_{1}, e_{1}\right\rangle=1,\left\langle e_{2}, e_{2}\right\rangle=1 \\
\left\langle e_{3}, e_{3}\right\rangle=-1\end{array}$ & $\begin{array}{c}\sin \alpha_{3} \neq 0, \\
\alpha_{1}+\alpha_{2} \neq 0, \\
\alpha_{1} \geqslant 0, \alpha_{2} \geqslant 0\end{array}$ \\
\hline $\mathcal{B}$ & $\begin{array}{c}{\left[e_{1}, e_{2}\right]=0,\left[e_{1}, e_{3}\right]=\alpha_{3} e_{1}-\alpha_{4} e_{2}} \\
{\left[e_{2}, e_{3}\right]=\alpha_{1} e_{1}+\alpha_{2} e_{2}}\end{array}$ & $\left\langle e_{2}, e_{2}\right\rangle=1,\left\langle e_{1}, e_{3}\right\rangle=-1$ & $\alpha_{2} \neq \alpha_{3}$ \\
\hline $\mathcal{C}_{1}$ & $\begin{array}{c}{\left[e_{1}, e_{2}\right]=0,\left[e_{1}, e_{3}\right]=\alpha_{3} e_{1}+\alpha_{1} e_{2}} \\
{\left[e_{2}, e_{3}\right]=\alpha_{1} e_{1}+\alpha_{2} e_{2}}\end{array}$ & \multirow{2}{*}{$\begin{array}{c}\left\langle e_{1}, e_{1}\right\rangle=1,\left\langle e_{2}, e_{2}\right\rangle=-1 \\
\left\langle e_{3}, e_{3}\right\rangle=1\end{array}$} & $\alpha_{2} \neq \alpha_{3}$ \\
\hline $\mathcal{C}_{2}$ & $\begin{array}{c}{\left[e_{1}, e_{2}\right]=0,\left[e_{1}, e_{3}\right]=\alpha_{2} e_{1}-\alpha_{3} e_{2},} \\
{\left[e_{2}, e_{3}\right]=\alpha_{1} e_{1}+\alpha_{2} e_{2}}\end{array}$ & & $\begin{array}{c}\alpha_{2} \neq 0 \\
\alpha_{1}+\alpha_{3} \neq 0\end{array}$ \\
\hline
\end{tabular}

- в случае $\mathcal{A}$ :

1. либо $\alpha_{1}=0, \alpha_{3}= \pm \pi / 2, V=\left( \pm \alpha_{2}, 0,0\right)$;

2. либо $\alpha_{1}=0, \alpha_{3}= \pm \pi / 2, V=\left(0,0, \mp \alpha_{2}\right)$;

3. либо $\alpha_{2}=0, \alpha_{3}= \pm \pi / 2, V=\left(0, \pm \alpha_{1}, 0\right)$;

4. либо $\alpha_{2}=0, \alpha_{3}= \pm \pi / 2, V=\left(0,0, \mp \alpha_{1}\right)$;

5. либо $\alpha_{1}=\alpha_{2}, V=\left(0,0,-\alpha_{1} \sin \left(\alpha_{3}\right)\right)$;

- в случае $\mathcal{B}: \alpha_{4}=0$ и
1. либо $V=\left(\alpha_{2}-\alpha_{3}, 0,0\right)$;
2. либо $V=\left(-\alpha_{2}, 0,0\right)$;

- в случае $\mathcal{C}_{1}: \alpha_{1}=0$ и

$$
\begin{aligned}
& \text { 1. либо } \alpha_{2}=0, V=\left(0,0, \alpha_{3}\right) ; \\
& \text { 2. либо } \alpha_{3}=0, V=\left(0,0, \alpha_{2}\right) ; \\
& \text { 3. либо } \alpha_{2}=0, V=\left(0, \pm \alpha_{3}, 0\right) .
\end{aligned}
$$

$B$ случаях $\mathcal{A}_{4}$ и $\mathcal{C}_{2}$ тензор кривизны не может быть равен нулю.

Доказательство. Рассмотрим доказательство теоремы для случая $\mathcal{A}_{3}$, для остальных случаев теоремы 1 доказательство аналогично.

В данном случае система уравнений $R=0$ имеет вид

$$
\begin{gathered}
\alpha_{1}^{2}-4\left(V^{3}\right)^{2}-4 V^{2}+8=0, \\
\alpha_{1}^{2}+4 V^{3}+4\left(V^{2}\right)^{2}-8=0, \\
\alpha_{1} V^{1}+2 V^{2} V^{3}+2 V^{2}-4=0, \\
\alpha_{1} V^{1}-2 V^{2} V^{3}-2 V^{3}+4=0, \\
\alpha_{1}^{2}+4\left(V^{1}\right)^{2}+4 V^{2}-4 V^{3}=0,
\end{gathered}
$$

$$
\begin{gathered}
\alpha_{1} V^{3}+2 V^{1} V^{2}-2 \alpha_{1}=0, \\
\alpha_{1} V^{2}+2 V^{1} V^{3}-2 \alpha_{1}=0, \\
\alpha_{1} V^{3}-2 V^{1} V^{2}+2 \alpha_{1}+2 V^{1}=0, \\
\alpha_{1} V^{2}-2 V^{1} V^{3}+2 \alpha_{1}+2 V^{1}=0 .
\end{gathered}
$$

Рассмотрим сумму седьмого и девятого уравнений и вычтем из них шестое и восьмое:

$$
\alpha_{1}\left(V^{2}-V^{3}\right)=0 .
$$

Если $\alpha_{1} \neq 0$, то $V^{2}=V^{3}$ и пятое уравнение примет вид $\alpha_{1}^{2}+4\left(V^{1}\right)^{2}=0$. Таким образом, обязательно $\alpha_{1}=0$. Система примет вид:

$$
\begin{gathered}
\left(V^{3}\right)^{2}+V^{2}-2=0, \\
V^{3}+\left(V^{2}\right)^{2}-2=0, \\
V^{2} V^{3}+V^{2}-2=0, \\
V^{2} V^{3}+V^{3}-2=0, \\
\left(V^{1}\right)^{2}+V^{2}-V^{3}=0, \\
V^{1} V^{2}=0, \\
V^{1} V^{3}=0, \\
V^{1} V^{2}-V^{1}=0, \\
V^{1} V^{3}-V^{1}=0 .
\end{gathered}
$$

Разность шестого и восьмого уравнений дает $V^{1}=0$, тогда из пятого получаем $V^{2}=V^{3}$. Система примет вид $\left(V^{2}\right)^{2}+V^{2}-2=0$ и будет иметь решения $V^{2}=1$ и $V^{2}=-2$. 
4. Заключение. В результате проведенных исследований построена математическая модель, которая позволяет вычислять компоненты тензора кривизны метрических групп Ли с метрической связностью с инвариантным векторным кручением. Кроме того, в работе доказана теорема об алгебраическом строении трехмерных метрических алгебр Ли с векторным кручением и нулевым тензором кривизны.

\section{Библиографический список}

1. Cartan E. Sur les variétés à connexion affine et la théorie de la relativité generalisée (deuxiéme partie) // Ann. Ecole Norm. Sup.1925. Vol. 42.

2. Muniraja G. Manifolds Admitting a Semi-Symmetric Metric Connection and a Generalization of Schur's Theorem // Int. J. Contemp. Math. Sci. 2008. Vol. 3. No 25.

3. Agricola I., Thier C. The Geodesics of Metric Connections with Vectorial Torsion // Annals of Global Analysis and Geometry. 2004. Vol. 26.

4. Murathan C., Özgür C. Riemannian manifolds with a semi-symmetric metric connection satisfying some semisymmetry conditions // Proceedings of the Estonian Academy of Sciences. 2008. Vol. 57. No 4.

5. Yilmaz H.B., Zengin F.Ö., Uysal. S.A. On a Semi Symmetric Metric Connection with a Special Condition on a Riemannian Manifold // European journal of pure and applied mathematics. 2011. Vol. 4. No 2.

6. Zengin F.Ö., Demirbağ S.A., Uysal. S.A., Yilmaz H.B. Some vector fields on a riemannian manifold with semi- symmetric metric connection // Bulletin of the Iranian Mathematical Society. 2012. Vol. 38. No 2.

7. Agricola I., Kraus M. Manifolds with vectorial torsion // Dierential Geometry and its Applications. 2016. Vol. 46.

8. Yano K. On semi-symmetric metric connection // Revue Roumame de Math. Pure et Appliquees. 1970. Vol. 15.

9. Sekigawa K. On some 3-dimensional curvature homogeneous spaces // Tensor N. S. 1977. Vol. 31.

10. Calvaruso G. Homogeneous structures on threedimensional Lorentzian manifolds // J. Geom. Phys. 2007. Vol. 57.

11. Milnor J. Curvature of left invariant metric on Lie groups // Advances in mathematics. 1976. Vol. 21.

12. Rodionov E.D., Slavskii V.V., Chibrikova L.N. Locally conformally homogeneous pseudo-Riemannian spaces // Siberian Advances in Mathematics. 2007. Vol. 17. No 3.

13. Cordero L.A., Parker P.E. Left-invariant Lorentzian metrics on 3-dimensional Lie groups // Rend. Mat. 1997. Vol. 17. 